\title{
Evolution des stratégies d'alimentation des élevages bovins dans le bassin d'approvisionnement en lait de la ville de Sikasso au Mali
}

\author{
D. Coulibaly ${ }^{1 *}$ C.H. Moulin ${ }^{2}$ R. Poccard-Chappuis ${ }^{3}$ \\ G. Morin ${ }^{2}$ S.I. Sidibé ${ }^{1}$ C. Corniaux ${ }^{3}$
}

Mots-clés

Bovin - Vache laitière - Troupeau Alimentation des animaux Evolution - Mali

\begin{abstract}
Résumé
Au cours des dernières décennies, I'alimentation des bovins est devenue la principale contrainte après la maîtrise des épizooties. Une étude a été conduite dans la zone périurbaine de Sikasso, ville moyenne en zone cotonnière du Mali, pour caractériser les évolutions de l'alimentation des troupeaux chez les éleveurs commercialisant du lait en ville. Des enquêtes rétrospectives ont été menées auprès de 27 responsables de troupeau pour cerner les changements de pratiques dans le long terme. Un suivi d'une année dans 14 troupeaux a permis de caractériser et quantifier les pratiques d'alimentation et la production de lait. Quatre stratégies d'alimentation des élevages bovins ont été distinguées : 1) troupeaux sédentaires, alimentés au pâturage, complémentation très faible ciblant plutôt les bœufs de labour et les jeunes ; 2) troupeaux sédentaires, alimentés au pâturage, faible soutien de la production laitière ; 3) troupeaux transhumants, avec allotement des laitières restant sur le terroir pour vendre du lait en ville, faible soutien de la production laitière ; 4) troupeaux au pâturage, éloignés de la ville ; laitières en ville, avec recours important aux concentrés. Le recours à une mobilité croissante est lié à la réduction de l'espace pastoral autour de la ville. La complémentation, fondée sur le stockage de biomasse fourragère et surtout l'achat de concentrés, a été facilité chez les agropasteurs par les avances sur les recettes du coton ou du lait mais a du mal à se pérenniser. L'utilisation des revenus du commerce a cependant permis à quelques éleveurs habitant en ville de développer un troupeau de laitières améliorées, avec une forte utilisation de la complémentation.
\end{abstract}

\section{INTRODUCTION}

Au Mali, comme dans la plupart des pays du Sahel, l'alimentation constitue aujourd'hui une des principales contraintes des productions bovines $(3,5,11,19,20,21,26)$. Cette alimentation est surtout fondée sur le pâturage de végétation spontanée et de résidus de cultures. La production laitière permise par l'élevage sur les pâturages est très fortement conditionnée par la disponibilité saisonnière et la valeur nutritive des fourrages $(4,33)$. Les zones de parcours

\footnotetext{
1. IER, BP 16, Sikasso, Mali.

2. SupAgro, UMR Elevage des ruminants en régions chaudes, Montpellier, France.

3. IER, Sikasso, Mali ; CIRAD, UR Systèmes d'élevage, Montpellier, France.

* Auteur pour la correspondance

Tél. : +22321621417; fax : +22321620349

E-mail : doubangolo@yahoo.fr, doubangolo.coulibaly@ier.ml
}

de végétation spontanée ont connu une dégradation sans précédent suite aux sècheresses des années 1970 et 80 avec, en conséquence, une descente des animaux au centre et au sud du pays (10). Pour faire face à la croissance démographique, l'extension des cultures grâce à la culture attelée, la mise en valeur des bas-fonds et des plaines inondables (riziculture, culture de contre saison) ont réduit fortement l'espace pastoral. Parallèlement, les effectifs du cheptel enregistrent les taux de croissance les plus élevés (épargne des revenus agricoles, sédentarisation des pasteurs transhumants), entraînant un surpâturage et une faible productivité du cheptel (1). La raréfaction des ressources pastorales amène les éleveurs à adopter de nouvelles pratiques et stratégies d'alimentation de leurs troupeaux.

Au-delà des contraintes agroécologiques, l'environnement socioéconomique (croissance démographique, urbanisation) a beaucoup contribué à l'évolution du secteur agricole. Le développement du marché urbain, l'appui technique, l'installation d'unités de 
transformation d'aliments, la création de laiteries sont les opportunités ayant favorisé l'adoption de nouvelles pratiques d'alimentation du bétail. La reprise des troupeaux par les agriculteurs aux éleveurs peuls a mis fin à la pratique de confiage et a modifié les relations entre ces acteurs. La pluriactivité nécessite chez les agropasteurs une réorganisation du travail du système de production.

Cet article analyse les stratégies développées par les éleveurs pour pallier les contraintes alimentaires croissantes. Il s'intéresse aux innovations paysannes et aux facteurs qui les favorisent ou les contraignent. L'étude a été menée dans la zone périurbaine de Sikasso, en zone cotonnière, où la densité du cheptel a fortement augmenté au cours des vingt dernières années.

\section{MATERIEL ET METHODES}

\section{Zone d'étude, potentialités, populations}

Le choix de la zone de Sikasso s'inscrivait dans le cadre d'un projet global sur l'élevage des bovins laitiers en zone périurbaine au Mali (8). Il s'est également appuyé sur les travaux des zonages agroécologiques et des systèmes de production réalisés en zone Mali-Sud $(2,7,36)$. La zone d'étude est située en zone soudanienne, avec des précipitations annuelles de $1200 \mathrm{~mm}$, bénéficiant ainsi des conditions climatiques favorables au développement des activités du secteur primaire (notamment l'agriculture, l'élevage). La contribution du secteur primaire au produit intérieur brut (PIB) est estimée à 20 p. 100 dont 46 p. 100 pour l'élevage (12). L'année est divisée en une saison des pluies, de mai à octobre, et une saison sèche découpée en saison sèche froide, de novembre à février, et en saison sèche chaude, de mars à avril. La température moyenne annuelle est environ de $26{ }^{\circ} \mathrm{C}$. Les paysages sont parcourus par de nombreux bas-fonds inondés pendant la saison des pluies et maintenant une importante humidité résiduelle dans les premiers mois de la saison sèche. Cette particularité agroécologique a offert des possibilités de diversification, aussi bien pour l'agriculture (riz inondé, maraîchage de contre-saison) que pour l'alimentation des bovins (pâturages de saison sèche). L'accès des troupeaux bovins aux bas-fonds est devenu difficile et parfois conflictuel avec la présence des cultures de contre-saison (7). Le système de culture coton - céréales est associé aux légumineuses. Les fourrages des résidus de culture sont stockés en partie par les éleveurs. Les champs récoltés représentant les parcours postculturaux sont exploités en vaine pâture. Les systèmes d'alimentation ont évolué et se sont adaptés à la disponibilité des ressources alimentaires.

Le choix s'est justifié également par la place socio-économique et l'urbanisation de la ville de Sikasso. Capitale de la troisième région, elle est située à environ $360 \mathrm{~km}$ au sud-est de Bamako et à $100 \mathrm{~km}$ des frontières de la Côte d'Ivoire et du Burkina Faso. La zone urbaine s'est agrandie et a atteint $940 \mathrm{~km}^{2}$, abritant 201292 habitants répartis entre la ville de Sikasso (135 484 habitants) et 59 villages sur un rayon de 30 kilomètres autour de la ville (13). La population est constituée par un brassage d'ethnies et de cultures du Mali et d'étrangers. Un doublement de la population est annoncé par les projections avec la croissance annuelle de 2,8 p. 100 à l'horizon 2025 (14). La croissance et la diversité de la population ont constitué, pour l'étude, une opportunité d'analyse du marché potentiel de consommateurs des produits agricoles, surtout d'animaux (lait, viande).

\section{Démarche d'étude}

Pour analyser les stratégies d'alimentation, deux pas de temps ont été pris en compte : le temps long (constitution du troupeau et trajectoire de l'exploitation) et le temps rond (cycles annuels de production). La démarche a été mise en œuvre dans le cadre de l'analyse des changements socio-techniques dans les systèmes de production laitière et la commercialisation du lait en zone périurbaine de Sikasso (8). L'étude a démarré par le recensement des éleveurs commercialisant du lait sur la ville de Sikasso. Un travail cartographique a permis de localiser les villages et les élevages commercialisant du lait pour approvisionner le marché urbain, et de délimiter ainsi le bassin laitier de Sikasso (figure 1). Après constitution de deux

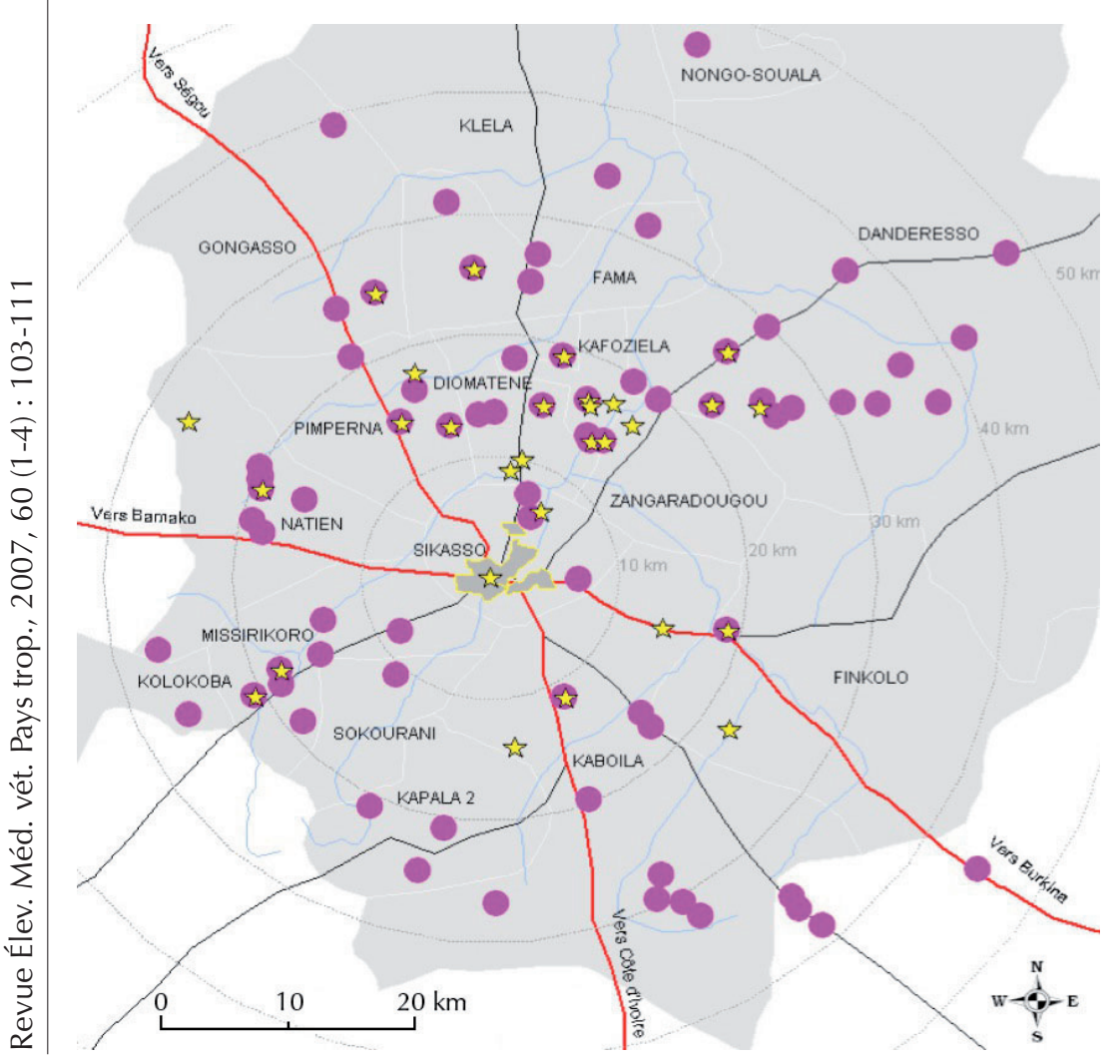

îs Eleveurs enquêtés

Villages commercialisant du lait vers Sikasso

Zone urbaine de Sikasso

Communes du bassin laitier sikassois

Isolignes de distance au centre-ville sikassois (écarts de $10 \mathrm{~km}$ )

Principaux cours d'eau

Principales pistes

Routes goudronnées

Figure 1 : localisation des unités de production de lait enquêtées. Dans un rayon de $50 \mathrm{~km}$ autour de Sikasso, des villages commercialisent du lait caillé vers le marché urbain. Ils délimitent le bassin laitier de Sikasso. Celui-ci est décalé vers le nord-est, où pâturages et points d'eau sont plus abondants. Les éleveurs enquêtés par le projet sont répartis uniformément dans ce bassin. 
échantillons, des enquêtes et des suivis zootechniques et économiques ont permis de retracer à la fois l'enchaînement saisonnier des pratiques d'alimentation et leurs évolutions au cours des années. Les prises de décisions des éleveurs ont ainsi pu être analysées.

\section{Echantillonnage}

Une enquête par questionnaire fermé a été réalisée en 2003 chez 60 éleveurs commercialisant du lait (sur les 153 recensés), répartis sur les différents axes d'approvisionnement en lait de la ville. A partir d'éléments structurels (surfaces cultivées, taille du cheptel), fonctionnels [disponible en aliments grossiers et en concentrés par unité de bétail tropical (UBT), pratiques sanitaires] et sur la commercialisation du lait, une stratification en cinq groupes a été réalisée (8). Pour la présente étude, un sous-échantillon (figure 2) de 27 éleveurs a été constitué, en prenant au hasard des éleveurs répartis par axe d'accès à la ville et par strate. Cette stratégie a assuré une représentation dans l'échantillon de la diversité en termes de systèmes d'élevage et de localisation (distance, accès) par rapport au marché urbain. Un second sous-échantillon a été constitué (14 parmi les 27) pour suivre le fonctionnement technique et économique de l'activité laitière sur une année.

\section{Enquêtes rétrospectives}

Les enquêtes rétrospectives se sont déroulées entre septembre et décembre 2005 pour retracer l'histoire de l'élevage de l'unité de production et plus particulièrement les changements dans la conduite de l'alimentation du troupeau. La démarche mise en œuvre a repris la méthode proposée par Moulin et coll. (29) pour analyser les transformations des pratiques d'éleveurs dans des petites régions françaises : territoire d'une coopérative laitière caprine dans les Cévennes (31) et élevage ovin transhumant hivernant dans la plaine de la Crau (30). Un guide méthodologique a été élaboré (28) et les enquêteurs ont été formés à conduire les entretiens. La démarche a consisté à faire raconter par le responsable du troupeau l'histoire de l'élevage bovin sur l'unité de production, depuis la constitution du troupeau par la famille ou au moins depuis l'accession à la tête du troupeau du responsable actuel. Les entretiens ont été organisés de la façon suivante : faire raconter l'organisation actuelle de l'élevage et de la commercialisation du lait (allotement, mobilité, types de ressources alimentaires, gestion et vente de lait); faire raconter les changements techniques survenus dans le long terme (constitution du cheptel, localisation et mobilité du troupeau, conduite) ; et faire s'exprimer l'éleveur sur les changements repérés pour en analyser les raisons et les conditions. Le traitement des informations s'est déroulé en deux étapes. Tout d'abord une chronique synthétique a été réalisée pour chaque unité de production (figure 3). Puis, deux analyses transversales ont été menées sur

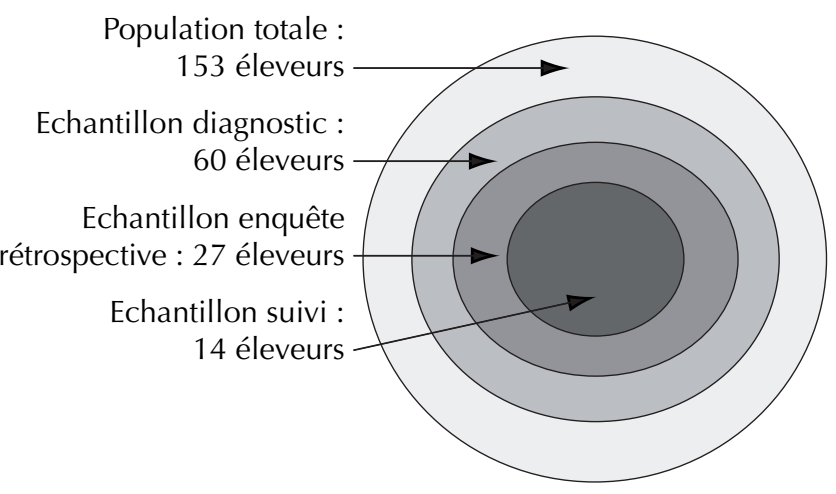

Figure 2 : échantillonnage des éleveurs laitiers autour de Sikasso.

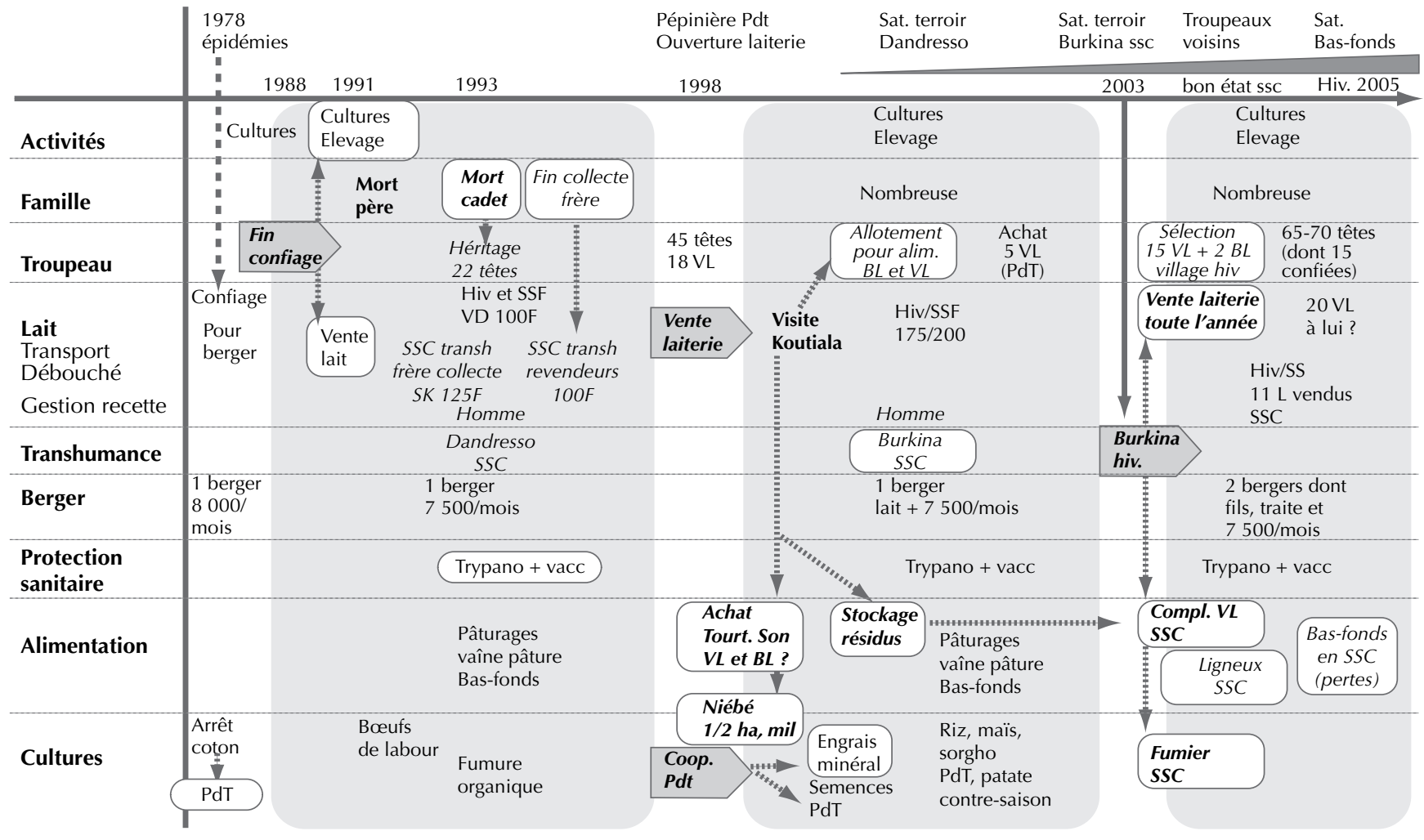

Figure 3 : exemple de chronique d'évolution.

SSC : saison sèche chaude ; transh : transhumance ; BL : bovin laitier ; VL : veau laitier ; PdT : pomme de terre ; Tourt. : tourteau. 
l'ensemble des chroniques : l'une pour caractériser la diversité des systèmes d'alimentation actuels, en considérant les pratiques de mobilité, d'allotement et de distribution d'aliments complémentaires ; l'autre pour dresser une typologie des trajectoires des unités de production, notamment par rapport à l'investissement dans l'élevage bovin et le développement de la commercialisation du lait.

\section{Suivi de troupeau}

Le suivi s'est déroulé entre août 2005 et juillet 2006 dans 14 unités de production, pour analyser sur un cycle annuel l'organisation de la production de lait et de sa commercialisation. Grâce à un passage mensuel, l'objectif a été notamment de pouvoir quantifier les stocks et les flux de matières (biomasse végétale, aliments du bétail, animaux, lait...) et les flux monétaires. La collecte des informations sur les pratiques d'alimentation du troupeau a porté sur les éléments suivants : la conduite du pâturage (zones de pâturage fréquentées, mobilité du troupeau, heure de sortie et retour du troupeau, nombre de bergers par jour) ; les stocks d'aliments (stock de départ, quantités mensuelles produites ou achetées, selon les types d'aliment, l'origine, le prix, la source de financement) ; et la distribution d'aliments (date de début et de fin de distribution de chaque type d'aliment, catégories animales bénéficiaires, états du stock...).

Le traitement a consisté à dresser un bilan alimentaire global à partir des aliments achetés (type, quantité, prix unitaire, coût du transport, période et rythme d'acquisition), des aliments produits (type, quantité, provenance, période), et de la distribution des aliments (période, catégorie animale ciblée).

\section{RESULTATS}

\section{Quatre familles de stratégies d'alimentation des troupeaux}

Quatre familles de stratégies d'alimentation ont été distinguées (tableau I). Dans les stratégies 1 et 2 , le troupeau était de taille très variable (10 à 120 têtes). Il passait toute l'année sur le terroir près de la ville, en exploitant les parcours naturels et les résidus de cultures laissés aux champs après les récoltes. Dans la stratégie 1 (troupeau sédentaire, au pâturage, complémentation très faible), les éleveurs distribuaient parfois des aliments complémentaires en saison sèche : des concentrés (son, graine de coton...) en très faibles quantités, rapportés au total des UBT du troupeau $(15 \mathrm{~kg} / \mathrm{UBT})$, et un peu de fanes de légumineuses. Ces apports étaient faits en saison sèche et ciblaient certaines catégories d'animaux, pour préparer les bœufs de labour avant la période de culture, et aider les jeunes et les animaux faibles à passer les périodes de soudure de la saison sèche. Les achats d'aliments concentrés étaient plutôt effectués sur la période de complémentation (janvier - avril, figure 4). Les pailles stockées étaient distribuées en vrac sous les animaux, visant plutôt la production de fumure organique pour l'entretien de la fertilité des champs en culture continue. Les deux éleveurs suivis exerçaient un taux moyen annuel de traite de 35 p. 100 de vaches traites (tableau I). La production laitière traite était très faible (en moyenne $70 \mathrm{~L} / v a c h e$ présente/ an) et concentrée sur la saison des pluies, la commercialisation du lait étant interrompue en saison sèche.

Dans la stratégie 2 (troupeau sédentaire, au pâturage, faible soutien de la production laitière), les pratiques d'alimentation étaient très semblables. Cependant l'effort de complémentation était plus soutenu (jusqu'à $200 \mathrm{~kg}$ de concentrés/UBT chez certains, soit 8 p. 100 des besoins théoriques annuels d'une UBT) et surtout il visait le troupeau dans son ensemble ou plus spécifiquement les laitières. Par ailleurs, les éleveurs ayant accès aux bas-fonds faisaient paitre les vaches laitières en prenant de gros risques d'accidents dans les puisards, cause de mort des veaux et parfois de vaches. La production laitière était un objectif plus marqué chez ces éleveurs exerçant des taux moyens annuels de traite variables (35-50 p. 100 de vaches traites), avec une production qui restait faible (100 L/vache présente/an) mais avec une commercialisation toute l'année.

Dans la stratégie 3 (troupeau transhumant, allotement des laitières, faible soutien de la production laitière), les troupeaux, de taille moyenne (70-100 têtes), effectuaient une transhumance de quelques mois. La transhumance de la saison sèche chaude avait lieu lorsque le pâturage manquait ou lorsque les stocks de résidus étaient épuisés. La contrainte d'abreuvement des gros troupeaux constituaient également un motif de départ en transhumance en cette période. La saturation des terroirs était le principal motif de la transhumance en saison des pluies, une fois les cultures installées. Ces éleveurs visaient eux aussi la commercialisation du lait toute l'année ; ils étaient donc amenés à séparer une partie des laitières pendant les périodes de transhumance pour les garder sur le village près de Sikasso. Elles étaient complémentées en saison sèche avec des concentrés, au même niveau que le groupe précédent ( 2 à 8 p. 100 des besoins théoriques annuels d'une UBT). Le reste de l'année, elles étaient conduites avec le reste du troupeau qui était revenu sur le village. La production laitière était un objectif prioritaire pour ces éleveurs, avec une commercialisation toute l'année, avec un taux moyen annuel de traite de 30 ou 70 p. 100 de vaches traites et une production de 93 ou $255 \mathrm{~L} /$ vache présente/an pour les deux éleveurs suivis dans cette stratégie.

Dans la stratégie 4 (troupeaux au pâturage, éloignés de la ville ; laitières en ville, avec fort recours aux concentrés), les cheptels étaient très importants (150 à 250 têtes). Ils étaient alors divisés en deux ou trois troupeaux éloignés toute l'année de Sikasso. Certains troupeaux revenaient seulement pour la période de vaine pâture afin de consommer en vaine pâture les résidus de culture laissés aux champs. Un petit troupeau (10 à 30 têtes), composé essentiellement de laitières, restait toute l'année à Sikasso, en zone périurbaine, parfois à l'intérieur même de la ville. Des échanges d'animaux étaient organisés entre le troupeau laitier et les autres troupeaux : une partie des vaches ayant mis bas étaient transférées dans le troupeau laitier, et les vaches taries et les veaux sevrés rejoignaient le troupeau d'élevage. Parfois seuls les veaux sevrés étaient transférés du troupeau laitier vers les autres troupeaux, sans qu'il y ait eu échange de vaches. Des inséminations ou des montes avec des taureaux de type laitier (Montbéliard notamment) ont permis de procréer des vaches métisses qui sont restées dans le troupeau laitier. Les trois éleveurs suivis mettant en œuvre cette stratégie avaient des conduites d'alimentation différentes, autorisant des taux moyens annuels de traite variables $(41,73$ et 45 p. 100 de vaches traites) et des niveaux différents de prélèvement de lait par vache présente du troupeau au cours de toute l'année (101, 601 et



Figure 4 : calendrier d'approvisionnement en aliments concentrés des unités de production. 
$797 \mathrm{~L} / v a c h e$ présente). Dans un premier cas, l'alimentation reposait sur le pâturage, avec des quantités importantes de concentrés distribués en saison sèche $(480 \mathrm{~kg})$. Dans un deuxième cas, le troupeau laitier pâturait toujours, mais recevait une très forte quantité de concentrés $(1320 \mathrm{~kg})$, distribués toute l'année, ainsi que des fourrages de bonne qualité pendant la saison sèche $(220 \mathrm{~kg}$ de fanes de légumineuses et de foin de brousse en fin de saison de pluies). Ces apports représentaient au total 62 p. 100 des besoins annuels d'une UBT, dont les concentrés constituaient 85 p. 100. Enfin, dans un troisième cas, les vaches étaient en stabulation permanente et toute l'alimentation distribuée à l'auge : $1740 \mathrm{~kg}$ de concentrés et $1980 \mathrm{~kg}$ de matière sèche (MS) de fourrages distribués par UBT. Ces apports en matière sèche représentaient 155 p. 100 des besoins théoriques annuels d'une UBT, constitués à 44 p. 100 de concentrés (le calcul de la consommation théorique se basait sur les besoins d'un bovin de $250 \mathrm{~kg}$ de poids vif à l'entretien, alors que les vaches assuraient ici une lactation, avec
797 litres traits par vache présente). Le stock d'aliments concentrés, composé majoritairement de son de céréales, est constitué en saison des pluies (juin - octobre), lorsque les prix étaient à leur plus bas niveau, l'offre étant supérieure à la demande. Pour la distribution de fourrages à l'auge, de l'herbe verte était fauchée quotidiennement sur les parcours et les jachères en saison des pluies. En saison sèche, la distribution d'un stock de paille de brousse, ramassée sur les parcours avant le passage des feux de brousse, était complétée par la fauche régulière des repousses d'herbe dans les bas-fonds.

\section{Chroniques des familles et évolutions de l'alimentation des troupeaux}

Les modalités d'alimentation des troupeaux sont issues d'un long processus d'évolution des systèmes d'élevage, depuis le lancement de l'activité d'élevage des familles (8). La stratégie 1 a été mise

\section{Tableau I}

Stratégies d'alimentation des bovins

\begin{tabular}{|c|c|c|c|c|c|c|c|c|c|}
\hline \multirow[t]{2}{*}{ Taille du troupeau (têtes) } & \multicolumn{9}{|c|}{ Stratégie d'alimentation } \\
\hline & \multicolumn{2}{|l|}{1} & \multicolumn{2}{|l|}{2} & \multicolumn{2}{|c|}{3} & \multicolumn{3}{|c|}{4} \\
\hline Nb. de bovins & $10-75$ & \multirow{2}{*}{\multicolumn{3}{|c|}{$\begin{array}{c}40-120 \\
(12)\end{array}$}} & \multicolumn{2}{|c|}{ 70-100 } & \multicolumn{3}{|c|}{$150-250$} \\
\hline (nb. d'enquêtes) & (8) & & & & \multicolumn{2}{|c|}{ (3) } & \multicolumn{3}{|c|}{ (4) } \\
\hline \multicolumn{10}{|c|}{ Organisation du troupeau et conduite } \\
\hline $\begin{array}{l}\text { Allotement } \\
\text { et mobilité }\end{array}$ & \multicolumn{3}{|c|}{$\begin{array}{l}1 \text { troupeau toute l'année } \\
\text { sur le terroir, près de la ville }\end{array}$} & & \multicolumn{2}{|c|}{$\begin{array}{c}\text { - } 1 \text { troupeau, avec } \\
\text { transhumance } \\
\text { (SSC et/ou SP) } \\
\text { - Laitières gardées sur } \\
\text { le terroir pendant } \\
\text { la transhumance }\end{array}$} & \multicolumn{3}{|c|}{$\begin{array}{c}-2 \text { à } 3 \text { troupeaux, } \\
\text { en transhumance lointaine } \\
-1 \text { troupeau de laitières } \\
\text { proche de la ville }\end{array}$} \\
\hline Pâturage & \multicolumn{6}{|c|}{ Toute l'année au pâturage } & \multicolumn{3}{|r|}{$\begin{array}{l}\text { Stabulation } \\
\text { permanente }\end{array}$} \\
\hline \multicolumn{10}{|c|}{ Distribution aliments complémentaires } \\
\hline Animaux ciblés & $\begin{array}{l}\text { Bovins laitie } \\
\text { jeunes, faibl }\end{array}$ & \multicolumn{5}{|c|}{ Troupeau, en particulier laitières } & \multicolumn{3}{|c|}{ Troupeau des laitières } \\
\hline $\begin{array}{l}\text { Distribution } \\
\text { fourrages }\end{array}$ & \multicolumn{2}{|c|}{ Faible (pailles, fanes) } & \multicolumn{2}{|c|}{ Forte } & \multicolumn{2}{|c|}{ Faible (pailles, } & & $\begin{array}{c}\text { Bons } \\
\text { fourrages, } \\
\text { en appoint }\end{array}$ & $\begin{array}{c}\text { Bons } \\
\text { fourrages } \\
\text { toute l'année }\end{array}$ \\
\hline $\begin{array}{l}\text { Apport } \\
\text { concentrés }\end{array}$ & \multicolumn{5}{|c|}{$\begin{array}{l}\text { Achat unique de grandes quantités } \\
\text { achat de faibles quantités étalées (nov. - avr.) }\end{array}$} & \multicolumn{4}{|c|}{$\begin{array}{l}\text { Achat de grandes quantités et constitution } \\
\text { de stocks en SP (juin - oct.) }\end{array}$} \\
\hline $\begin{array}{l}\text { Distribution } \\
\text { concentrés }\end{array}$ & \multicolumn{2}{|c|}{ Faible } & \multicolumn{4}{|c|}{ Moyenne } & Forte & \multicolumn{2}{|c|}{ Très forte } \\
\hline \multicolumn{10}{|c|}{ Données quantitatives observées pendant le suivi : prélèvement de lait selon les stratégies d'alimentation } \\
\hline $\begin{array}{l}\text { Lait/vache } \\
\text { présente/an (L) (n) }\end{array}$ & $70(2)$ & & $70-110(7)$ & & $93(1)$ & $254(1)$ & $101(1)$ & $601(1)$ & $797(1)$ \\
\hline $\begin{array}{l}\text { Taux moyen annuel } \\
\text { de traite }(\%)(\mathrm{n})\end{array}$ & $35(2)$ & & $35-50(7)$ & & $30(1)$ & $70(1)$ & $41(1)$ & $73(1)$ & $45(1)$ \\
\hline $\begin{array}{l}\text { Concentrés } \\
\text { (kg/UBT/an) (n) }\end{array}$ & $0-14(2)$ & $0-35(4)$ & $60(2)$ & $200(1)$ & $50(1)$ & $160(1)$ & $480(1)$ & $1320(1)$ & $1740(1)$ \\
\hline $\begin{array}{l}\text { Fourrages } \\
\text { (kg MS/UBT/an) (n) }\end{array}$ & $\begin{array}{c}0(1) \\
620(1)\end{array}$ & $\begin{array}{c}40-75(3) \\
590(1)\end{array}$ & $10-160(2)$ & $520(1)$ & $5(1)$ & $16(1)$ & $5(1)$ & $220(1)$ & $1980(1)$ \\
\hline
\end{tabular}

SSC : saison sèche chaude ; SP : saison des pluies ; MS : matière sèche ; UBT : unité de bétail tropical

Stratégie 1 : troupeaux sédentaires, au pâturage, complémentation très faible.

Stratégie 2 : troupeaux sédentaires, au pâturage, faible soutien de la production laitière.

Stratégie 3 : troupeaux transhumants, allotement des laitières, faible soutien de la production laitière.

Stratégie 4 : troupeaux au pâturage, éloignés de la ville; laitières en ville, avec recours important aux concentrés. 
en œuvre par des familles d'agroéleveurs, pour lesquelles les revenus des cultures étaient plus importants que ceux du troupeau. Ces familles ont développé un troupeau bovin par investissement de revenus de l'agriculture ou de travail salarié à l'extérieur. Le troupeau était surtout un capital et une source de travail et de fumier pour l'agriculture. La commercialisation du lait est récente, depuis la fin des années 1990 ou le début des années 2000. Seuls les surplus de lait, après autoconsommation, étaient commercialisés en saison des pluies, la vente étant interrompue en saison sèche chaude, voire même dès la saison sèche froide. La production laitière n'était pas un objectif prioritaire et les pratiques d'alimentation ne visaient pas à soutenir cette production. Le ramassage de résidus de culture pouvait être important chez certains (jusqu'à $600 \mathrm{~kg}$ de MS de paille/UBT) mais orienté vers la production de fumure organique.

Les stratégies 2 et 3 , avec faible soutien de la production par la complémentation, avec des troupeaux sédentaires ou transhumants, se rencontraient dans deux types de familles. Tout d'abord dans des familles d'agroéleveurs (famille de grande taille, jusqu'à plusieurs dizaines de personnes, avec beaucoup de surfaces cultivées) ayant développé un troupeau bovin en capitalisant les revenus des cultures. Cet investissement dans l'élevage bovin s'est fait pour certains avant même l'introduction de la culture du coton et de l'utilisation de bœufs de labour. La production laitière était plus importante que dans le cas précédent. Ces stratégies ont également été mises en œuvre par des familles d'agropasteurs de petite taille (une dizaine de personnes au maximum). L'activité d'élevage était plus importante que l'agriculture, les quelques cultures permettant une autosuffisance en céréales. La production laitière du troupeau bovin permettait de procurer des rentrées d'argent régulières.

Selon la localisation géographique (plus ou moins éloignée de Sikasso), l'importance des bas-fonds dans le terroir village, la proximité ou non de zones de brousse résiduelles pour le pâturage en saison des pluies, ces familles devaient entreprendre des mouvements de transhumances (stratégie 3) pour alimenter leurs troupeaux, ce qui les amenait à séparer les laitières du troupeau une partie de l'année. Dans la stratégie 2, des mouvements de transhumance ont été observés depuis peu, certaines années, en fin de saison sèche chaude. Les apports de concentrés étaient très variables, en fonction de la qualité des zones de pâturage accessibles.

La stratégie 4, avec un grand troupeau extensif éloigné et un troupeau de laitières en ville, était développée par des commerçants peuls. Quittant le nord du pays, notamment lors des sécheresses des années 1970 et 80, ils ont pratiqué le commerce (tissu, bétail) et investi les revenus de ces activités dans l'élevage bovin. Lorsque le troupeau devenait trop grand (plus de 80-100 têtes), il ne pouvait pas rester autour de Sikasso, par manque de place pour pâturer. Certains de ces commerçants ont alors gardé un troupeau de laitières dans la ville ou en zone périurbaine proche, avec des investissements dans l'amélioration génétique (insémination artificielle pour produire des vaches métisses) et la mise en œuvre d'une conduite fondée sur l'utilisation importante d'intrants (concentrés) et de travail (récolte de biomasse en brousse et de résidus de cultures). La commercialisation du lait se faisait directement en ville.

\section{DISCUSSION}

\section{Intérêts et limites de l'échantillonnage et du recueil de données}

L'échantillonnage des unités observées a reposé sur une stratification, fondée sur les systèmes de production mis en œuvre et leur localisation sur les axes d'accès à la ville. La diversité des situations a ainsi été bien représentée dans les deux échantillons emboîtés (27 unités de production enquêtées et 14 suivies). En revanche, ces échantillons n'étaient pas représentatifs de la population et il n'a pas été possible d'évaluer le poids des différentes trajectoires ou des stratégies d'alimentation dans la population totale des élevages commercialisant du lait. L'échantillonnage réalisé sur la base des éleveurs vendant du lait sur le marché urbain excluait les unités de production ne vendant pas du lait au moment des enquêtes. Les causes des échecs des pratiques d'alimentation chez ces éleveurs ne peuvent pas être répertoriées par l'analyse à long terme. Cependant certaines causes d'abandon de pratiques ont pu être identifiées. Ainsi, dans certaines trajectoires des unités de production, les éleveurs ont arrêté puis repris la complémentation des animaux mais aussi la vente de lait.

Les deux dispositifs de recueil de données étaient très complémentaires. La méthode d'enquêtes rétrospectives a permis d'accéder à l'histoire d'un grand nombre d'unités de production, en peu de temps (deux à trois mois). Elle s'est montrée applicable à l'analyse des systèmes d'élevage extensifs ou semi-intensifs sous les tropiques, après deux applications dans l'analyse des systèmes d'élevage de bovins laitiers en zone périurbaine de Sikasso (8) et de Ségou (27). Les données recueillies étaient essentiellement qualitatives, faisant plutôt appel à la mémoire de l'éleveur. Les recoupements des dires recueillis lors des deux entretiens auprès de chaque éleveur ont permis de construire des informations fiables. A partir des récits du chef de famille ou du responsable du troupeau, l'histoire des unités de production et l'évolution des pratiques d'alimentation des troupeaux ont pu être retracées. L'analyse des trajectoires à l'échelle d'une zone d'élevage, puis à l'échelle de l'ensemble du bassin, a permis de caractériser les changements et de repérer les dates et les événements ayant marqué l'histoire de l'évolution des systèmes d'élevage. La méthode d'enquête a beaucoup contribué à l'analyse de l'histoire des systèmes d'élevage dont la seule source était la mémoire du chef d'exploitation, responsable du troupeau, ou le berger. Les éléments des chroniques peuvent être utilisés dans d'autres études pour créer des bases d'informations ou comme clés typologiques.

Les informations du suivi obtenues étaient d'abord quantitatives et ont été utilisées pour caractériser les pratiques et évaluer les efforts d'alimentation des éleveurs. Elles ont en outre contribué à l'établissement des calendriers de conduite du troupeau, et d'approvisionnement et de distribution d'aliments. La confiance acquise avec l'éleveur lors des passages répétés a permis d'améliorer la qualité et la fiabilité des informations. Ce dispositif est donc très précieux, permettant de quantifier, grâce à des observations, des données qui ne sont obtenues que par des déclarations d'éleveurs dans le cadre des enquêtes. Cependant, il nécessite des moyens importants et n'a pu être mis en place que sur un nombre limité de troupeaux, rendant crucial le choix des unités suivies. Le suivi était aussi l'occasion de recueillir des données qualitatives, complémentaires de celles obtenues par les enquêtes rétrospectives. Il a permis à l'éleveur de s'exprimer sur ses choix techniques actuels, au moment où il les mettait en œuvre, en les replaçant dans le cadre de leur mise en œuvre concrète, selon les conditions du moment (organisation de la décision, du travail, climat...). Les enquêtes rétrospectives ont permis d'accéder à des justifications a posteriori des choix réalisés, fondées sur la relecture du passé faite par chaque individu.

La combinaison des deux dispositifs avait l'avantage de faciliter l'identification et la caractérisation des pratiques innovantes et des changements majeurs opérés. Les contraintes et les solutions de développement de l'élevage et de pratiques durables d'alimentation ont été aisément identifiées. Par exemple, l'ouverture de la laiterie a été à l'origine de la complémentation (crédit aliments concentrés), de l'enrichissement de la paille à l'urée, de cultures fourragères dans les bas-fonds, et de la vente du lait par les hommes avec un retour sur le troupeau (remboursement du prêt aliment et intrants vétérinaires sur les recettes du lait). La mise en ouvre de cette démarche 
dans d'autres systèmes de production peut contribuer à l'avancée des méthodes de recherche de la zootechnie - systèmes pour analyser les systèmes de production dans toute leur complexité.

\section{Diversité des stratégies d'alimentation du troupeau bovin}

Les quatre stratégies d'alimentation identifiées ont été distinguées selon l'utilisation du pâturage du terroir et des résidus de culture et de concentrés en appoint (stratégies 1 et 2), la pratique de la transhumance proche, l'allotement des vaches laitières gardées sur le terroir (stratégie 3), la transhumance lointaine et le maintien d'un troupeau de laitières proche de la ville avec distribution importante d'aliments complémentaires (stratégie 4).

Les crises climatiques et agraires déclenchées depuis les années 1970 et $80(3,5)$ ont engendré une concentration des effectifs de bovins au sud $(2,26)$. Les stratégies d'adaptation des systèmes de production, notamment d'alimentation des élevages, aux changements de l'environnement avaient déjà été décrites en Afrique subsaharienne (18, 34, 35). La taille actuelle du troupeau a été atteinte par accumulation des revenus agricoles, en particulier le coton $(2,8)$, et la capitalisation des économies du commerce ou des salaires (8) dans le bétail. Elle détermine dans une large mesure l'organisation et la conduite du troupeau, et aussi les quantités et la distribution des aliments complémentaires. L'allotement et la mobilité sont apparus aux yeux des éleveurs comme une stratégie pour faire face à la raréfaction progressive des parcours naturels. La mobilité a conduit dans certains cas à la délocalisation d'une partie du troupeau (stratégie 4). Cette pratique laisse encore de beaux jours à l'objectif toujours prioritaire de capitalisation des agriculteurs, des pasteurs peuls sédentarisés et des commerçants peuls. La transhumance se révèle une pratique flexible $(29,30)$, adaptée à la saturation des terroirs et répondant à la capitalisation du bétail. Cependant, elle pose des problèmes d'organisation (main d'œuvre, perte de lait, de fumier) incompatibles à l'intégration agriculture - élevage. La «pastoralisation » des troupeaux sédentaires des agriculteurs ne favorise guère un développement durable des systèmes de production.

La distribution des aliments complémentaires devient sélective quand l'effectif est élevé. Elle cible les bœufs de labours, les jeunes, les animaux faibles en saison sèche chaude (stratégie 1), le troupeau (fourrages utilisés plutôt en litière pour produire le fumier) ou en particulier les laitières (concentrés) pour les stratégies 2 et 3 . Enfin, tout le troupeau des laitières reçoit du bon fourrage et des quantités élevées de concentrés toute l'année au retour du pâturage ou en stabulation (stratégie 4). La disponibilité et la qualité des ressources fourragères sont à l'origine de la croissance en dents de scies des jeunes, de perte de poids des adultes $(2,3,4,5,33)$ et des carences alimentaires (32). L'amélioration des productions animales nécessite donc une amélioration de l'alimentation du cheptel en produisant plus d'aliments sur l'exploitation (stratégie 4).

En plus de la taille du troupeau, l'organisation du travail (allocation de la main d'œuvre entre les activités agricoles et d'élevage) pèse énormément dans la conduite du troupeau et en particulier le stockage des résidus de culture. La répartition de la main d'œuvre disponible se fait au détriment des activités d'élevage (8). La prise en compte de l'organisation du travail est aussi essentielle en exploitation d'élevage et dans l'utilisation du territoire $(24,25)$ pour résoudre le problème de travail dans des systèmes d'élevage améliorés en France. Dans les systèmes de production mixtes agriculture - élevage, le potentiel de biomasse végétale est encore faiblement valorisé dans l'alimentation des bovins à cause des pointes de travail des opérations de récolte et de ramassage des résidus de culture $(8,9,11)$. Le ramassage et le transport des fourrages reviennent aux enfants (stratégies 1,2,3) et aux salariés (stratégie 4). La vaine pâture, le plus souvent collective, profite peu à l'alimentation du troupeau de la famille, surtout si elle est de taille réduite en compétition avec les transhumants revenus sur le terroir pour exploiter les parcours postculturaux.

Enfin, l'organisation et la conduite du troupeau relèvent du chef de famille ou du responsable du troupeau, mais les tâches sont réalisées par d'autres acteurs (membres de la famille, berger). Les systèmes d'élevage sont donc pilotés par un système multiacteurs, dont les prises de décisions de chaque acteur sont déterminantes dans la gestion du troupeau (8). Des études conduites dans des systèmes d'élevage similaires à Ségou au Mali par Morin et coll. (27), au Sénégal par Corniaux et coll. (6), et dans d'autres pays du Sud par Lhoste (22) montrent toute l'importance du poids de chaque acteur dans l'organisation sociotechnique de l'élevage. Le berger joue un rôle capital dans la conduite du troupeau, de par ses connaissances du pâturage au cours des saisons de l'année. Le chef de famille décide les achats d'aliments concentrés en fonction de la trésorerie des revenus agricoles ou du lait (stratégies 1,2), des recettes du lait (stratégie 3) et des revenus du commerce pour constituer des stocks de concentrés (stratégie 4).

\section{Facteurs du changement des pratiques d'alimentation du troupeau bovin}

Les chroniques construites ont donné la possibilité de repérer les facteurs de changement. La saturation des terroirs est ressortie dans les chroniques, comme facteur à l'origine des pratiques d'allotement, de transhumance ou encore de distribution de fourrages ligneux chez les agriculteurs et les commerçants peuls ayant capitalisé dans les bovins $(8,27)$. Le développement de la culture attelée et les aménagements des bas-fonds ont accru la pression sur les espaces pastoraux et les ressources fourragères $(2,7,19,20)$. Les gros troupeaux effectuent dès lors la transhumance de saison sèche et de saison des pluies, rendant difficile l'intégration agriculture - élevage (10, 15, 17, 22). Chez ces agropasteurs, l'adoption du paquet technique de stockage des résidus de culture, pour la production de fumure et d'alimentation du troupeau promue par la Compagnie malienne pour le développement des textiles (Cmdt) ou la laiterie, présente des trajectoires différents (stratégies 1,2,3). Lhoste (22), en parlant du faible niveau d'intégration de l'agriculture et de l'élevage, a conclu à des systèmes techniques d'élevage et de culture juxtaposés au sein d'un même système de production. L'arrêt du crédit d'aliments de la Cmdt ou de l'appui de la laiterie a entrainé l'abandon de la complémentation ou sa diminution, mais il est survenu à différentes périodes dans les chroniques individuelles d'évolution des unités de production. Les éleveurs ont perdu la faveur du paiement des intrants sur la recette du coton ou du lait. En plus des facteurs du milieu, les pesanteurs socio-économiques interviennent beaucoup dans les transformations des pratiques d'alimentation du troupeau (cultures fourragères, achat des aliments concentrés). Le stockage des résidus de culture ou l'achat des concentrés sont ainsi apparus dans les pratiques d'alimentation. Le financement est cité par les éleveurs comme une contrainte majeure à l'alimentation et, de façon générale, à l'activité d'élevage. L'insuffisance des stocks d'aliments complémentaires a déclenché le départ en transhumance des gros troupeaux.

Ces résultats ont confirmé l'hypothèse que le problème d'alimentation était la principale contrainte de l'élevage. Les parcours naturels sont de plus en plus résiduels dans le bassin d'approvisionnement de la ville et les disponibles en aliments par UBT sont largement inférieurs aux besoins d'entretien des troupeaux. Ceci concorde avec les travaux de nombreux auteurs $(5,9,11,15,19,21,26)$ qui concluent que les systèmes d'alimentation du cheptel bovin sont fondés sur les parcours naturels. Une production animale n'est envisageable qu'à partir des apports d'aliments complémentaires (fourrages, concentrés), en ciblant un lot spécifique ou quelques animaux. 


\section{CONCLUSION}

Les systèmes alimentaires des troupeaux sédentaires, fondés exclusivement sur les parcours naturels, sont passés à des systèmes transhumants, des systèmes avec apports d'aliments en appoint au pâturage. Les changements des systèmes d'alimentation ont résulté des changements socio-techniques, et notamment des facteurs agroécologiques et climatiques. La conjonction de ces facteurs a accru la pression sur les ressources pastorales. Les éleveurs, face au problème grandissant d'alimentation des bovins, ont développé des stratégies flexibles d'organisation et de conduite du troupeau. L'allotement du troupeau facilite la circulation des animaux dans les terroirs de plus en plus occupés par les cultures, même en saison sèche, avec la présence des cultures de contre-saison dans les bas-fonds. La transhumance, voire la délocalisation, répond à la capitalisation, objectif principal des éleveurs propriétaires de gros troupeaux. L'intégration agriculture - élevage a beaucoup contribué à la transformation des pratiques d'alimentation du troupeau par la production de fourrages et l'utilisation des résidus. Les systèmes de crédits d'aliments et la formation des éleveurs aux techniques d'alimentation (Cmdt, laiterie) ont profondément modifié la conduite extensive des troupeaux. La distribution ciblée des aliments concentrés est à l'origine de la création de noyaux de vaches laitières, puis de troupeaux de laitières localisés en ville ou proches de la ville. Les stratégies d'alimentation reposant sur un allotement des laitières (stratégies 3 et 4) présentent des perspectives réelles pour soutenir un développement laitier pour l'approvisionnement des marchés urbains. Ces stratégies passent par la valorisation des ressources fourragères naturelles ou cultivées et des aliments concentrés mais nécessitent un recours important à l'achat d'intrants et à la main d'œuvre pour la récolte de biomasse végétale.

Sur le plan des textes réglementaires, l'application de la charte pastorale et des conventions locales pourra apporter des améliorations dans la gestion de l'espace pastoral (définition des zones de pâturage, création ou redynamisation des comités de gestion, respect des textes en vigueur). Concernant les techniques de production, des améliorations pourraient être envisagées sur les pratiques de valorisation des résidus de culture (stockage, traitement mécanique, chimique), de production et conservation des fourrages de bonne qualité sur l'exploitation (culture fourragère, ensilage), et de distribution d'aliments complémentaires en recherchant une meilleure complémentarité entre les fourrages et les concentrés. Cela implique la maitrise des techniques de production et de conservation, la connaissance des circuits d'approvisionnement, et la gestion des stocks d'aliments complémentaires. Ces axes d'intervention permettraient de lever les contraintes d'alimentation, notamment responsables de la saisonnalité laitière, rendant davantage vulnérables les productions locales aux importations.

\section{BIBLIOGRAPHIE}

1. BONNET B., 1988. Etude de l'élevage dans le développement des zones cotonnières : le Mali. Mémoire Ing., Cnearc, Montpellier, France, p. 110.

2. BOSMA R., BENGALY K., TRAORE M., ROELEVELD A., 1996. L'élevage en voie d'intensification : synthèse de la recherche sur les ruminants dans les exploitations agricoles mixte au Mali-Sud. Amsterdam, Netherlands, Royal Institute of the Tropics, p. 202.

3. BREMAN H., RIDDER N., 1991. Manuel sur les pâturages des pays sahéliens. Paris, France, Karthala, 485 p.

4. BREMAN H., SISSOKO K., 1998. L'intensification agricole au Sahel. Paris, France, Karthala, 996 p.

5. BREMAN H., TRAORE M., 1987. Analyse des conditions de l'élevage et propositions de politiques et de programmes. Wageningen, Netherlands, CABO, $234 \mathrm{p}$.
6. CORNIAUX C., DUTEURTRE G., DIEYE P.N., POCCARD-CHAPUIS R., 2005. Les minilaiteries comme modèle d'organisation des filières laitières en Afrique de l'Ouest : succès et limites. Revue Elev. Méd. vét. Pays trop., $\mathbf{5 8}: 237-243$.

7. COULIBALY D., 1998. Caractérisation des systèmes de production dans la zone périurbaine de Sikasso. Mém. Institut polytechnique rural de formation et de recherche appliquée, IPR/Ifra, Katibougou, Mali, p. 74.

8. COULIBALY D., 2008. Changements socio-techniques dans les systèmes de production laitière et commercialisation du lait en zone péri-urbaine de Sikasso, Mali. Thèse Doct. Zootechenie des systèmes d'élevage, Agro-Paristech, France, p. 399. http://pastel.paristech.org/5012

9. COULIBALY D., POCCARD-CHAPUIS R., MOULIN C.H., 2006. Typologie des exploitations d'élevage de la zone périurbaine de Sikasso approvisionnant en lait la ville. In : Atelier régional Vers de nouvelles politiques laitières en Afrique de l'Ouest, Bamako, Mali, 29 mai - 2 juin 2006.

10. D'AQUINO P., LHOSTE P., LE MASSON A., 1995. Interaction des systèmes de production d'élevage et l'environnement : systèmes de production mixtes agriculture pluviale et élevage en zone humide et subhumide d'Afrique. Montpellier, France, Cirad-emvt, p. 103.

11. DEMBELE N'F., 1995. Etude économique de la disponibilité et de I'utilisation des suppléments dans I'alimentation des bovins au Mali : étude de cas des éleveurs du cercle de Koutiala. Thèse Doct. AgroEconomie, Isfra, Bamako, Mali, p. 151.

12. DNSI, 2005. Annuaire statistique. Bamako, Mali, Dnsi.

13. DNUH, 2005. Schéma directeur d'urbanisation de la ville de Sikasso et environs. Rapport final. Sikasso, Mali, Dnuh, $115 \mathrm{p}$.

14. DRPSIAP, 1998. Schéma directeur d'urbanisation de la ville de Sikasso et environs. Rapport annuel. Bamako, Mali, Drpsiap.

15. JOSHUA R., 1999. La longue saison sèche : interaction agricultureélevage dans le sud du Mali. Madison, WI, US, University of Wisconsin, p. 29.

16. KAUFMANN J.C., 1996. L'entretien compréhensif. Paris, France, Nathan.

17. KEBE D., 1989. Les relations agriculture-élevage et le devenir des systèmes de production Fonsébougou Sud-Mali. Mém. DEA, Ensa, Montpellier, France, p. 66.

18. KOUSSOU M.O., 2008. Dynamique des innovations dans le secteur de l'élevage au Tchad : le cas de la filière laitière de N'Djamena. Thèse Doct., Agro ParisTech, France, 243 p.

19. LELOUP S., TRAORE M., 1990. La situation fourragère dans le sudest du Mali. Régions Cmdt de Sikasso et de Koutiala, tome I. Amsterdam, Netherlands, KIT, 94 p.

20. LELOUP S., TRAORE M., 1991. La situation fourragère dans le sud-est du Mali. Région Cmdt de San, tome II. Amsterdam, Netherlands, KIT, 71 p.

21. LE MASSON A., 1996. Programme national de la production laitière au Mali. Les systèmes d'élevage à vocation laitière : contraintes, propositions. Montpellier, France, Cirad-emvt, 95 p. (Rapport nº 96 030)

22. LHOSTE P., 2004. L'analyse des transformations des systèmes d'élevage dans les pays du sud : questions et perspectives. In : Chia E., Dedieu B., Moulin C.H., Tichit M., éds, actes séminaire Transformation des pratiques techniques et flexibilité des systèmes d'élevage, Inra-SAD, Montpellier, France, 15-16 mars 2007.

23. LHOSTE P., DOLLE V., ROUSEAU J., SOLTNER D., 1993. Manuel de zootechnie des régions chaudes. Les systèmes d'élevage. Paris, France, ministère de la Coopération, 288 p. (Coll. Manuel et précis d'élevage)

24. MADELRIEUX S., 2004. Ronde des saisons, vie des troupeaux et labeur des hommes. Modélisation de l'organisation du travail en exploitation d'élevage herbivore au cours d'une année. Thèse Doct., INAPG, Paris, France.

25. MADELRIEUX S., DEDIEU B., DOBREMEZ L., 2002. Modifications de I'utilisation du territoire lorsque les éleveurs cherchent à résoudre leurs problèmes de travail. Fourrages, 172 : 355-368.

26. Ministère de I'Agriculture, de l'Elevage et de l'Environnement, 1992. Schéma directeur du secteur développement rural : stratégies de développement, volume II. Bamako, Mali, Maee.

27. MORIN G., COULIBALY D., CORNIAUX C., POCCARD-CHAPPUIS R., SIDIBE S.I., MOULIN C.H., 2007. Dynamiques des unités de production laitière dans le bassin d'approvisionnement de la ville de Ségou au Mali. Revue Elev. Méd. vét. Pays trop., 60. 
28. MOULIN C.H., COULIBALY D., POCCARD-CHAPUIS R., CORNIAUX C., 2005. Guide méthodologique pour I'analyse des changements de l'unité de production commercialisant du lait en zone urbaine au Mali : mise au point d'un protocole d'enquête rétrospective et de traitement de l'information. Montpellier, France, Cirad-emvt, p. 30.

29. MOULIN C.H., INGRAND S., LASSEUR J., MADELRIEUX S., NAPOLEONE M., PLUVINAGE J., THENARD V., 2004. Comprendre et analyser les changements d'organisation et de conduite de l'élevage dans un ensemble d'exploitation : propositions méthodologiques. Paris, France, Inra, p. 12

30. MOULIN C.H., PLUVINAGE J., BOCQUIER F., 2004. Les relations entre agrandissement des troupeaux et changements de conduite : exemple des élevages ovins allaitant en Crau. Rencontres Rech. Ruminants, 11 : 145-148.

31. NAPOLEONE M., BOUTONNET J.P., 2004. AOC Pélardon : du compromis vers l'émergence d'actions collectives. Dynamiques de systèmes de production et des stratégies de commercialisation. In : Sémin. Systèmes de productions agricoles, performances, évolutions, perspectives, Lille, France, 18-19 nov. 2004, 11 p.
32. OUOLOGEM B., TOURE I., COULIBALY D., POCCARD-CHAPUIS R., SANGARE M.I., COULIBALY L., FANE A., 2007. Mise au point de méthodes de lutte contre le syndrome paralytique des bovins dans la région de Sikasso. Bamako, Mali, Institut d'économie rurale.

33. PENNING DE VRIES F.W.T., DJITEYE M.A., 1991. La productivité des pâturages sahéliens. Une étude des sols, des végétations et de I'exploitation de cette ressource naturelle. Wageningen, Netherlands, Pudoc, 525 p. (Agricutural Reseach Reports 918)

34. PRESTON T.R., 1987. Adaptation des systèmes d'élevage aux ressources alimentaires disponibles dans les pays tropicaux. Wageningen, Netherlands, CTA.

35. PRESTON T.R., 1998. Le développement des systèmes de production laitière sous les tropiques. Wageningen, Netherlands, CTA, 71 p.

36. TOGOLA M., DEMBELE S., 1998. Plan stratégique de la recherche agricole 1999-2005. Sikasso, Mali, Crra, p. 201.

Accepté le 02.02.2010

\section{Summary}

Coulibaly D., Moulin C.H., Poccard-Chappuis R., Morin G., Sidibé S.I., Corniaux C. Changes in the Feeding Strategies of Cattle Herds in the Dairy Basin of the City of Sikasso in Mali

In the last decades, cattle feeding became the main constraint after disease management. A study was carried out in the periurban area of Sikasso, an average size city in the cotton area of Mali, to characterize changes in the feeding strategies of breeders selling dairy products in town. A retrospective survey involving 27 herd managers was conducted to identify changes in practices on the long term. Fourteen herds were monitored during one year to characterize and quantify feeding practices and milk production. Four feeding strategies of animal husbandry were distinguished: 1) sedentary grazing herds, with low supplementation, mainly for draft, and young animals; 2) sedentary grazing herds, with low supplementation of dairy cows; 3) transhumant herds, with dairy cows staying near the town for the sale of milk, and low supplementation of dairy cows; 4) herds grazing far away from town, with dairy cows kept in town and fed high levels of concentrates. Herd mobility resulted from a decrease in grazing lands around the town. Supplementation, based on forage biomass reserves and above all purchased concentrates, was made available to agropastors through an advance on the benefits of cotton or milk sales, but it was hard to maintain on the long term. Profits from the trade enabled a few breeders in town to develop a herd of improved dairy cows fed large amounts of concentrates.

Keywords: Cattle - Dairy cow - Herd - Animal feeding Evolution - Mali.

\section{Resumen}

Coulibaly D., Moulin C.H., Poccard-Chappuis R., Morin G., Sidibé S.I., Corniaux C. Evolución de las estrategias de alimentación de las criaderos bovinos en la meseta de abastecimiento de leche de la ciudad de Sikasso en Mali

En el curso de las últimas décadas, la alimentación de los bovinos se ha transformado en el principal obstáculo, luego del control de las epizootias. Se llevó a cabo un estudio en la zona peri urbana de Sikasso, ciudad mediana en la zona algodonera de Mali, para caracterizar las evoluciones en la alimentación de los hatos de los criadores que comercializan leche en la ciudad. Se Ilevaron a cabo encuestas retrospectivas con 27 responsables de hato, para cernir los cambios de prácticas a largo plazo. Un seguimiento de un año de 14 hatos permitió la caracterización y la cuantificación de las prácticas de alimentación y la producción de leche. Se distinguieron quatro estrategias de alimentación en los criaderos bovinos: 1) hato sedentario, alimentado con pasto, complemento bajo, orientado sobre todo hacia los bueyes de trabajo y los jóvenes; 2) hato sedentario, alimentado con pasto, poco apoyo a la producción lechera; 3) hato nómada, con mantenimiento de lecheras en el territorio para vender la leche en la ciudad, bajo apoyo a la producción lechera; 4) hatos de pastoreo, alejados de la ciudad; lecheras en la ciudad, con fuerte acceso a concentrados. El recurso a una movilidad creciente está ligado a la reducción del espacio pastoril alrededor de la ciudad. La suplementación, fundada sobre el almacenamiento de biomasa forrajera y sobre todo la compra de concentrados, ha sido facilitada para los pastores agrícolas mediante de adelantos sobre las ganancias del algodón o de la leche, pero tarda en perennizarse. El uso de los ingresos del comercio a sin embargo permitido a algunos criadores que viven en la ciudad desarrollar un hato de lecheras mejoradas, con una fuerte utilización de suplementos.

Palabras clave: Ganado bovino - Vaca lechera - Hato Alimentación de los animales - Evolución - Malí. 